\title{
A two-dimensional finite element model of front surface current flow in cells under non-uniform, concentrated illumination
}

\author{
A. Mellor, J.L. Domenech-Garret *, D. Chemisana, J.I. Rosell \\ Departament de Medi Ambient i C.S., University of Lleida, Av. Alcalde Rovira Roure 191, E25198, Spain
}

\begin{abstract}
A two-dimensional finite element model of current flow in the front surface of a PV cell is presented. In order to validate this model we perform an experimental test. Later, particular attention is paid to the effects of non-uniform illumination in the finger direction which is typical in a linear concentrator system. Fill factor, open circuit voltage and efficiency are shown to decrease with increasing degree of non-uniform illumination. It is shown that these detrimental effects can be mitigated significantly by reoptimization of the number of front surface metallization fingers to suit the degree of non-uniformity. The behavior of current flow in the front surface of a cell operating at open circuit voltage under non-uniform illumination is discussed in detail.
\end{abstract}

Keywords: Solar cells; Non-uniform concentrated illumination; Fill factor efficiency; Finite elements model; Short circuit current; Open circuit voltage

\section{Introduction}

PV concentrator systems provide an effective means of reducing the cost of electricity production by reduction of the amount of silicon required. PV cells operating under concentrated illumination are subject to high current densities. Consequently detailed attention must be paid to series resistance within the cell, particularly when optimizing the front contact grid to make an effective compromise between shading and front surface resistive losses.

The main contributions to series resistance in the front surface of a solar cell are the emitter sheet resistance, the contact resistance between the emitter and the metal finger, the finger resistance and the busbar resistance (Cole et al., 2006). Of course for a given element of generated current, the distance travelled laterally through the emitter, finger, and busbar, and hence the resistive loss, will be dependent on the spatial coordinate of the part of the junction at which that element is generated. The total resistive loss is the sum of the resistive losses to which current elements produced at all points on the junction are subject.

Models have been devised using the above considerations, which make the simplifying assumption that current generation is uniform across the cell (Cole et al., 2006). However, this approach ignores the so called 'distributed diode effect', which can be summarized as follows. The lateral resistances in the cell lead to a voltage drop across the cell surface causing different points on the cell surface operate at different voltages and therefore produce different current densities as determined by the IV curve. Under concentrated illumination this effect is significant as current densities and therefore resulting voltage drops are high. Any accurate model of series resistance in concentrator cells must therefore seek to allow the current density produced at any spatial junction element to vary according to the voltage at which that element is operating.

Illumination profiles produced by concentrator systems are generally non-uniform (Johnston, 1998; Luque et al., 1998; Coventry et al., 2004). Non-uniform illumination 
has been shown experimentally (Franklin and Coventry, 2003 ) and by means of simulation (Luque et al., 1998) to negatively effect the performance of a solar cell by reduction of fill factor and open circuit voltage. Franklin et al. devised a two-dimensional model taking into account the distributed diode effect and the effects of non-uniform illumination (Franklin and Coventry, 2003), but make the simplifying assumption that current flow in the emitter is perpendicular to the finger. As the degree of non-uniformity is increased, this assumption becomes less valid as potential gradients parallel to the fingers can cause components of current flow in this direction. A three-dimensional, two diode model for GaAs solar cells taking many factors into account has also been devised by Galiana et al. (2005).

This paper presents a two-dimensional finite element model of current distribution in the front surface of the cell which takes into account the distributed diode effect, and further reduces the assumptions of Franklin and Coventry (2003) by not restricting current in the emitter to flowing perpendicular to the fingers. The model is used to demonstrate the reduction in fill factor and open circuit voltage as a result of non-uniform illumination, and further to demonstrate that the reduction in fill factor can be mitigated by reoptimization of the front contact grid to suit the given degree of non-uniformity.

\section{Model}

\subsection{Selection of domain}

The whole cell can be considered as consisting of a number of identical finger elements as shown in Fig. 1; this is chosen to be the domain in which the simulations are made. In fact, one could reduce the domain size by a further factor of four by considering the lines of symmetry which run in the $\mathrm{x}$ and $y$ directions. However, simulating the entire emitter section instead of a quarter of it has the advantage that identical regions are reproduced, and therefore can be used to check for any anomalies in the numerical method, particularly around troublesome edge regions. It also reduces the edge effects in the emitter.

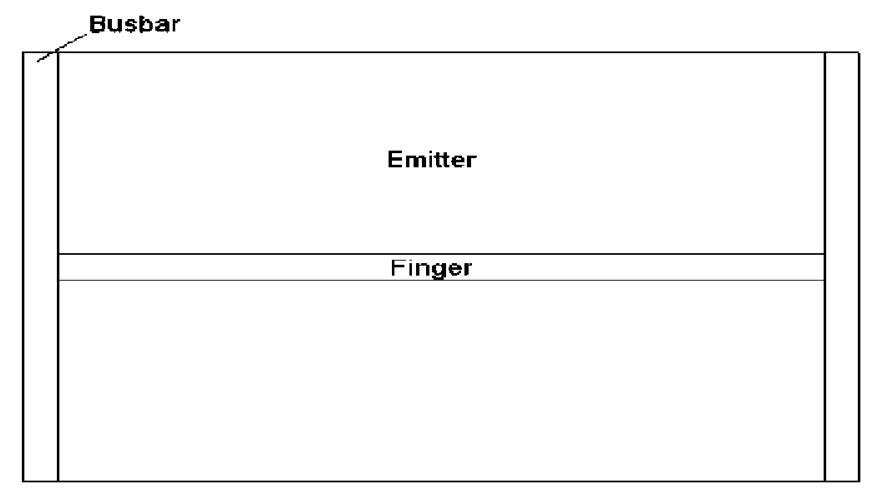

Fig. 1. The cell element to be modeled.

\subsection{Illumination profile}

Illumination profiles incident on cells in linear concentrators are generally most non-uniform in the finger direction, typically resembling a Gaussian function on this axis (Johnston, 1998). Non-uniformity may also exist to a lesser extent in the busbar direction due to a variety of factors (Coventry, 2005), however this is not considered in this paper. Movement of the illumination profile across the cell due to tracking misalignment is also ignored.

The model takes the $\mathrm{x}$ dependence of the illumination intensity incident on the cell, where the $x$ axis is the finger direction with origin at the middle of the cell, to be:

$G(x)=G_{0} A_{0} \exp \left(-\frac{x^{2}}{2 S_{0}^{2}}\right)$

where $G_{0}$ is the mean illumination across the cell, $S_{0}$ controls the width of the curve and can be related to the Full Width at Half the Maximum ( $F W H M$ ) by:

$F W H M=2 \sqrt{2 \ln (2)} S_{0}$

$A_{0}$ is the normalization factor which ensures the mean irradiance across the cell is $G_{0}$, and is equivalent to the ratio of peak to mean irradiation, it is given by:

$A_{0}=\frac{W}{\sqrt{2 \pi} S_{0} \operatorname{erf}\left(\frac{W}{2 \sqrt{2} S_{0}}\right)}$

where $\operatorname{erf}($ ) is the error function and $W$ the cell width in the finger direction. In our models the irradiance is normalized over the active area of the cell, not including the busbars, and hence $W$ is taken to be the width of this region. Normalising over the whole cell would result short circuit current increasing with increased non-uniform illumination because busbar shading decreases as the illumination becomes more central.

\subsection{Partial differential equation (PDE) formulation}

As we are dealing with DC current flow in a conductive medium, the PDE to be solved in the domain is the continuity equation

$-\nabla \cdot\left(\sigma \nabla V-J^{e}\right)=Q_{j}$

where $V$ is the electric potential, $J^{e}$ is the current density, $Q_{j}$ is the current source term and $\sigma$ is the sheet conductivity of the material defined as

$\sigma=1 / R_{\text {sheet }}$

where $R_{\text {sheet }}$ is the sheet resistance in ohms per square.

When dealing with the emitter, the sheet resistance is a common parameter and is experimentally obtainable. When dealing with the fingers, the parameter most easily determined by experiment is the resistance per unit length $R_{p . u l}$. For the purposes of defining the PDE over the whole domain, a theoretical sheet resistance for the finger can be defined as

$R_{\text {sheet }}=R_{p . u . l} \times w$ 
where $w$ is the finger width. A similar consideration can be made for the busbars.

The current source $Q_{j}$ can be interpreted as the current density which is generated by the diode. The one diode model is used because according to the literature (van der Heide et al., 2005) more accurate values can be found for all parameters for such a model. In the case of the two diode model the strong interdependence of the different variables spoils the accuracy in the fit procedure with experimental data. Taking into account photogenerated current, recombination, and shunts, the current source term in the illuminated emitter region can be expressed as (Luque and Hegedus, 2003):

$$
\begin{aligned}
Q_{j}= & C_{1} G+C_{2} T^{3} \exp \left(\frac{-E_{g}}{k_{b} T}\right)\left[\exp \left(\frac{q_{e} V_{j}}{n k_{b} T}\right)-1\right] \\
& +C_{3} V_{j}
\end{aligned}
$$

and in the dark finger and busbar regions can be expressed as

$Q_{j}=C_{2} T^{3} \exp \left(\frac{-E_{g}}{k_{b} T}\right)\left[\exp \left(\frac{q_{e} V_{j}}{n k_{b} T}\right)-1\right]+C_{3} V_{j}$

where $G$ is the illumination with profile described in Section $2.1, T$ is the cell temperature, $V_{j}$ is the junction electric potential, $q_{e}$ is the electron charge, $k_{b}$ is the Boltzmann constant, $E_{g}$ is the bandgap energy, $n$ is the diode ideality factor, and $C_{1}, C_{2}$ and $C_{3}$ are coefficients specific to a given cell, whose origins are described in Rosell and Ibañez (2006). It must be pointed out that this model has been developed basing on a silicon monocrystalline solar cell, nevertheless in principle it could be applied to other cell types.

\subsection{Boundary conditions}

There are three types of boundary condition to be considered in this problem. These are:

Interface condition : $-n_{b} \cdot\left(J_{1}-J_{2}\right)=0$

Electric insulation : $n_{b} \cdot J=0$

Busbar electric potential : $V=V_{\text {cell }}$

where $n_{b}$ is the unit normal to the boundary, $J_{1}$ and $J_{2}$ are the current density vectors at the boundary of adjacent media, and $J$ is the current density vector at the external boundary.

The interface condition is applied to all internal boundaries to ensure continuity of current at interfaces between different media. The longitudinal outside edges of the busbars and external boundaries of the emitter section (actually lines of symmetry within the cell) are considered to be electrically insulating. The ends of the busbars are considered to be connected to the external load and should therefore have electric potential equal to the cell operating voltage $V_{\text {cell }}$, an input parameter.

\subsection{Solution found using the Finite Element Method}

The current and voltage distribution, and in particular current output at the busbars, is found by the solution of

(4), applying (7) and (8) in the appropriate subdomains, subject to conditions (9)-(11) at the appropriate boundaries. The solution is obtained using the Finite Element Method, implemented using the commercially available software package $\mathrm{ComSol}^{\odot}$, this software also allows the internal running of SPICE models, furthermore each model can be checked by running it with different numerical solvers and meshes in order to achieve the desired numerical accuracy. The theory behind the Finite Element Method is both complex and well documented. An explanation is not, therefore, included in this paper, but may be found in Zienkiewicz et al. (2005) as well as numerable other sources.

\subsection{Validation of the model: experimental test}

First, in order to validate our model we compare the numerical values obtained by means of the model with experimental results. A silicon monocrystalline ASE solar cell, with a known set of $C_{1}, C_{2}, C_{3}$ experimental coefficients obtained from measures taken under homogeneous radiation, was used. The cell parameters are summarized in Table 1.

Table 1

Input parameters used in the validation of the model.

Cell geometry and resistivities

Cell length (busbar direction)

Cell width (finger direction)

Busbar width

Finger width

Finger resistivity

$9.7 \mathrm{~cm}$

$4.4 \mathrm{~cm}$

$2 \mathrm{~mm}$

$15 \mu \mathrm{m}$

$65.42 \mathrm{n} \Omega \mathrm{m}$

Operation conditions

Temperature

$298.16 \mathrm{~K}$

Mean Illumination Intensity $6850 \mathrm{Wm}^{-2}$

Diode equation parameters

$C_{1}$

$C_{2}$

$C_{3}$

Ideality factor $n$

$E_{g}$

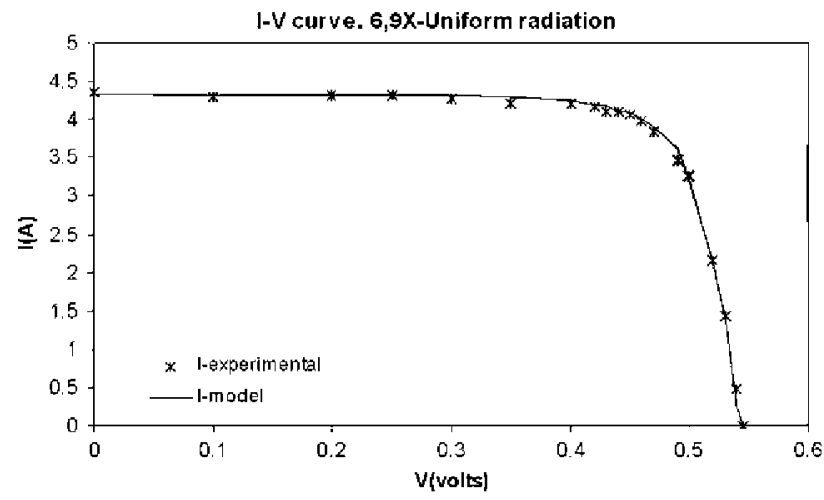

Fig. 2. Experimental and modeled curve for 6,9X-uniform radiation.

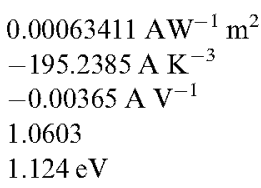

$00063411 \mathrm{AW}^{-1} \mathrm{~m}^{2}$ 1.0603 


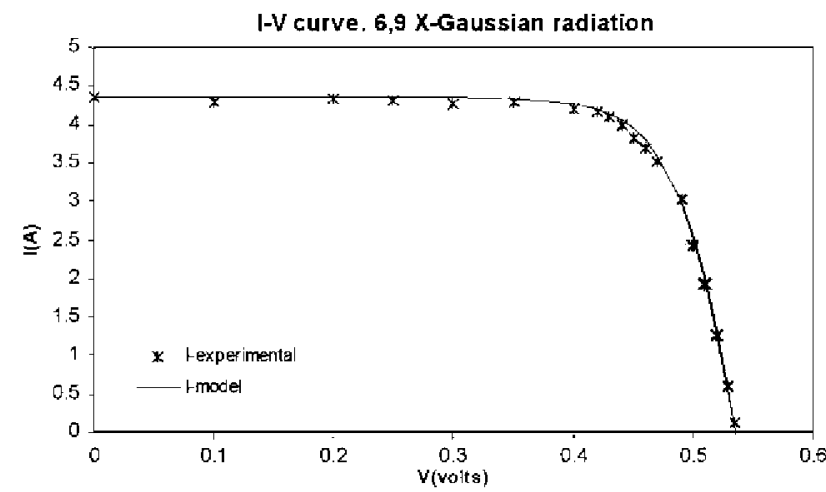

Fig. 3. Experimental and modeled curve for 6,9X-Gaussian radiation.

Experimental data was obtained using an indoor device at the Research Center in Applied Energy (CREA), University of Lleida. It consists of a continuous light concentrator device yielding 6,9 suns concentration. The ASE cells are illuminated uniformly and using Gaussian filters to work under non-uniform patterns. A cooling system is used to maintain a temperature of $298.2 \mathrm{~K}$. The experimental points are stored with a standard Computer DAQ system.

The results of the experiment vs. model can be seen in Fig. 2 for a uniform radiation profile and in Fig. 3 for a gaussian radiation profile with $S_{0}=5 \mathrm{~mm}$.

\section{Results for 12 suns}

Input parameters: The input parameters used in the model are given in Table 1.The cell length (in the busbar direction), and width (in the finger direction) are chosen

Table 2

Input parameters used in all simulations and IV characteristics for cell simulated under 12 suns uniform illumination.

\begin{tabular}{ll}
\hline Cell geometry and resistivities & \\
Cell length (busbar direction) & $10.6 \mathrm{~cm}$ \\
Cell width (finger direction) & $4.8 \mathrm{~cm}$ \\
Busbar width & $2 \mathrm{~mm}$ \\
Finger width & $35 \mu \mathrm{m}$ \\
Finger resistance per unit length & $0.3 \Omega \mathrm{cm}^{-1}$ \\
Emitter sheet resistance & $100 \Omega / \square$ \\
Operation conditions & \\
Temperature & $320 \mathrm{~K}$ \\
Mean illumination Intensity & $12,000 \mathrm{Wm}^{-2}$ \\
Diode equation parameters & \\
$C_{1}$ & $0.39444 \mathrm{AW}^{-1}$ \\
$C_{2}$ & $-11,739 \mathrm{Am}^{-2} \mathrm{~K}^{-3}$ \\
$C_{3}$ & $-0.83584 \mathrm{Am}^{-2} \mathrm{~V}^{-1}$ \\
Ideality factor $n$ & 1.0603 \\
$E_{g}$ & $1.124 \mathrm{eV}$ \\
$I V$ characteristics for cell simulated under 12 suns uniform illumination \\
$I_{\text {sc }}$ & $20.79 \mathrm{~A}$ \\
$V_{\mathrm{oc}}$ & $0.65 \mathrm{~V}$ \\
FF & 0.79 \\
Efficiency & $19.25 \%$ \\
\hline
\end{tabular}

to be within an appropriate range for cells operating in a linear concentrator. Further cell parameters, namely finger and busbar widths and resistances per unit length and emitter sheet resistance are preliminary data measured from experimental Laser Grooved Buried Contact (LGBC) cells developed at the New and Renewable Energy Company (NaREC), in Northumberland, UK.

The purpose of this study is not to simulate a specific existing cell, but to examine generically the effects of nonuniform illumination on PV cell behavior. The parameters $C_{1}, C_{2}$, and $C_{3}$ are described in Section 2.3. In the case of the coefficients chosen, the characteristics produced at 12 suns uniform illumination are shown in Table 2.

All modeled results in this section were obtained using the parameters stated in Table 2 . The temperature distribution across the cell was assumed to be uniform. All illumination profiles for non-uniform illumination are Gaussian curves as described in Section 2.2. Maintaining the nomenclature of Franklin and Coventry (2003), the parameter used to define the degree of non-uniformity is the peak illumination ratio, defined as the ratio between the peak illumination at the cell centre and the mean illumination across the cell. This is mathematically equivalent to the normalization factor $A_{0}$ discussed in Section 2.2. The total radiation absorbed by the active area of the cell is the same in all illumination profiles, the mean illumination being 12 suns.

In house algorithms have been written to find accurately the open circuit voltage $\left(V_{\mathrm{OC}}\right)$ and maximum power point $\left(P_{\max }\right)$ by comparison of results from simulations made at adjacent voltage steps.

\subsection{Optimization of the number of front contact fingers for a range of peak illumination ratios}

When designing the front contact metallization pattern for a solar cell, the number of front contact metallization fingers must by chosen so as to minimize the sum of shading losses and ohmic resistive losses which respectively increase and decrease with increasing number of fingers.

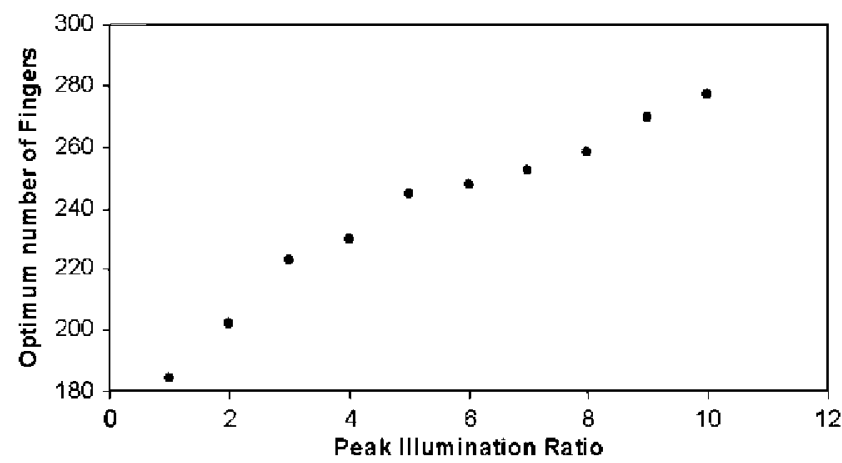

Fig. 4. The number of fingers found to optimize the cell efficiency for Gaussian illumination profiles with a range of peak illumination ratios. In each case the mean illumination intensity across the active area of the cell is 12 suns. 

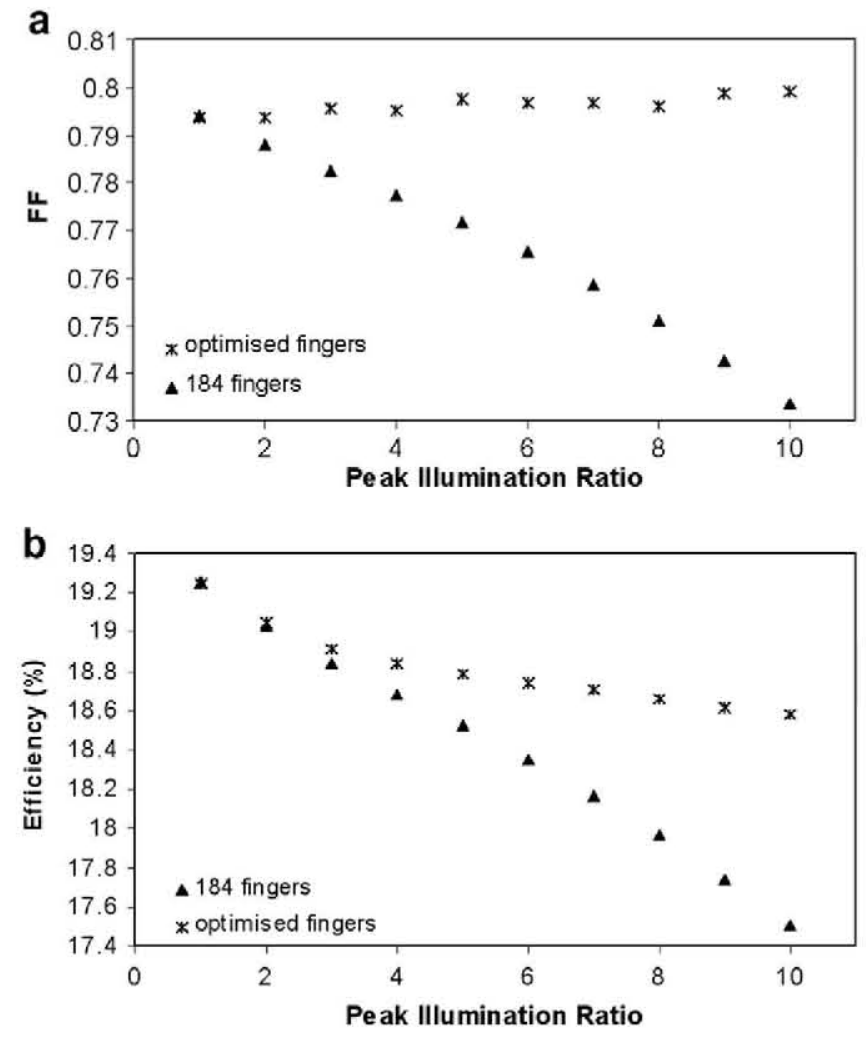

Fig. 5. Fill factors (a) and cell efficiencies (b) of cells simulated under Gaussian illumination profiles with a range of peak illumination ratios. The triangles are data points for cells with 184 fingers. The stars are for cells with finger number optimized for the illumination profile under which they are operating as shown in Fig. 2.
It is interesting to see what effect non-uniformity of illumination has on the number of fingers needed to optimize the front contact pattern. Algorithms have been written to simulate a range of cell geometries, and hence optimize the number of fingers in the front contact grid for any given degree of non-uniformity. The results are shown in Fig. 4, a plot of the optimum number of fingers against the peak illumination ratio. The optimum number of fingers increases notably with increasing peak illumination ratio, increasing from 184 fingers under uniform illumination, to 227 fingers under non-uniform illumination with a peak illumination ratio of 10 .

\subsection{Fill factor and efficiency}

The red points in Fig. 5a and $\mathrm{b}$ show respectively the variation of fill factor (FF) and efficiency with increasing peak illumination ratio for a cell with 184 fingers found to be optimum for uniform illumination. The decrease in fill factor suggests an increase in effective series resistance. This can be attributed to the distribution of current densities in the emitter, which is shown as a three-dimensional plot in Fig. 4 for a single finger section of a cell under Gaussian non-uniform illumination. Of course most of the current generated in the cell is localized around the highly illuminated area, leading to huge resistive losses in this region.

The blue points in Fig. 5a and b show respectively the variation of fill factor and efficiency with increasing peak illumination ratio for cells optimized for the illumination profile under which they are operating, as described in

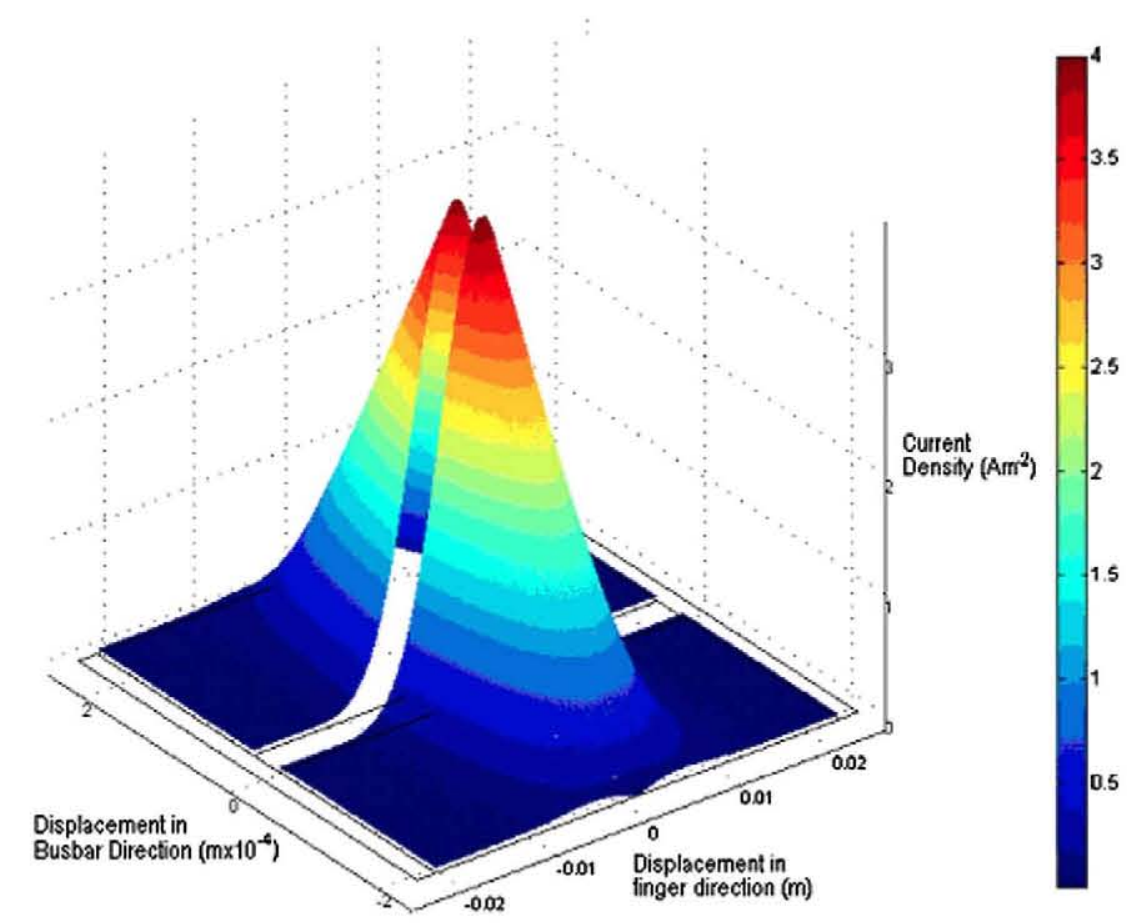

Fig. 6. Three-dimensional plot of current density in the emitter of a single finger element. Both height and colour data represent current density. Outline of busbars and finger are shown beneath the plot in black. Current density in the fingers and busbars is not plotted. 
Section 3.1. It can be seen that increasing the number of fingers on the front surface of a cell under non-uniform illumination mitigates the increase in series resistance significantly, making considerable improvement to the cell fill factor and efficiency.

\subsection{Short circuit current $\left(I_{s c}\right)$ and open circuit voltage $\left(V_{o c}\right)$}

Concerning the $I_{\mathrm{sc}}$ behavior at different non-uniform illumination profiles, our simulations performed at 12 suns show no significant variation across the entire range of tested profiles; this is consistent with the results found in Franklin and Coventry (2003).

Fig. 7 shows the variation open circuit voltage $\left(\mathrm{V}_{\mathrm{oc}}\right)$ with increasing peak illumination ratio. Again the red

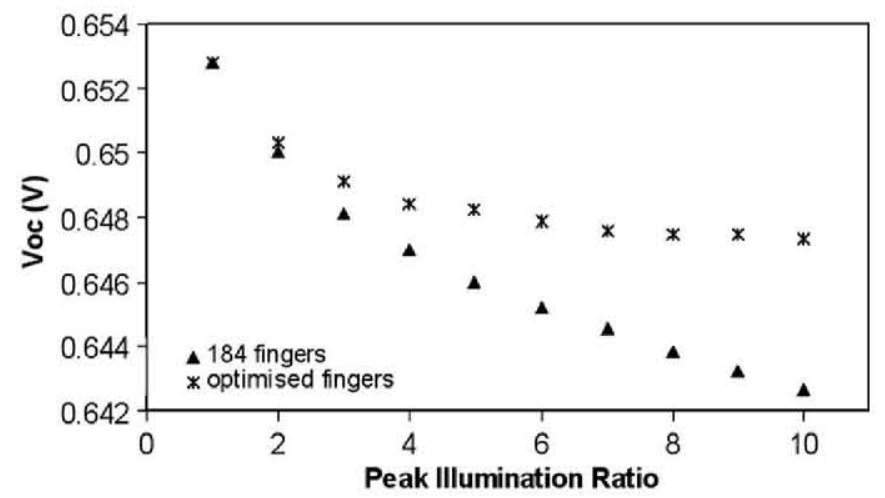

Fig. 7. Open circuit voltages of cells simulated under Gaussian illumination profiles with a range of peak illumination ratios. The triangles are data points for cells with 184 fingers. The stars are for cells with finger number optimized for the illumination profile under which they are operating as shown in Fig. 2. points indicate values obtained from simulated cells with 184 fingers, and the blue points indicate values obtained for cell geometries optimized for the illumination profile under which they are operating. The $V_{\mathrm{oc}}$ is shown to decrease with increasing illumination non-uniformity, though the drop is less severe than that observed in the fill factor. Again the effect is reduced by reoptimization of the front contact pattern.

The behavior of the cell close to open circuit voltage under non-uniform illumination is extremely interesting. Although the cell generates no net current there are internal currents flowing within the cell. The red lines in Fig. 8 are streamlines of current flow in the cell operating at open circuit voltage under non-uniform illumination. It can be seen that current generated in the highly illuminated central region of the cell flows via the fingers to the darker edge regions where it is absorbed. As shown in Fig. 9, a cross sectional plot of the spatial variance of the voltage in the finger direction, the regions from which and to which the current flows correspond to voltage maxima and minima respectively. Indeed this must be the case, as current flow must coincide with negative potential gradients. At first glance it would seem counter intuitive for forward current to be generated in the high voltage region and reverse current to be generated in the low voltage region as the former would be expected to be operating above the open circuit voltage and the later operating below. To understand this behavior one must consider that there is not only spatial dependence of the junction voltage and hence operating point on the IV curve, but also of the IV curve itself due to the spatial dependence of the illumination. That is to say that any given point on the emitter can be considered as having its own local IV curve, depen-

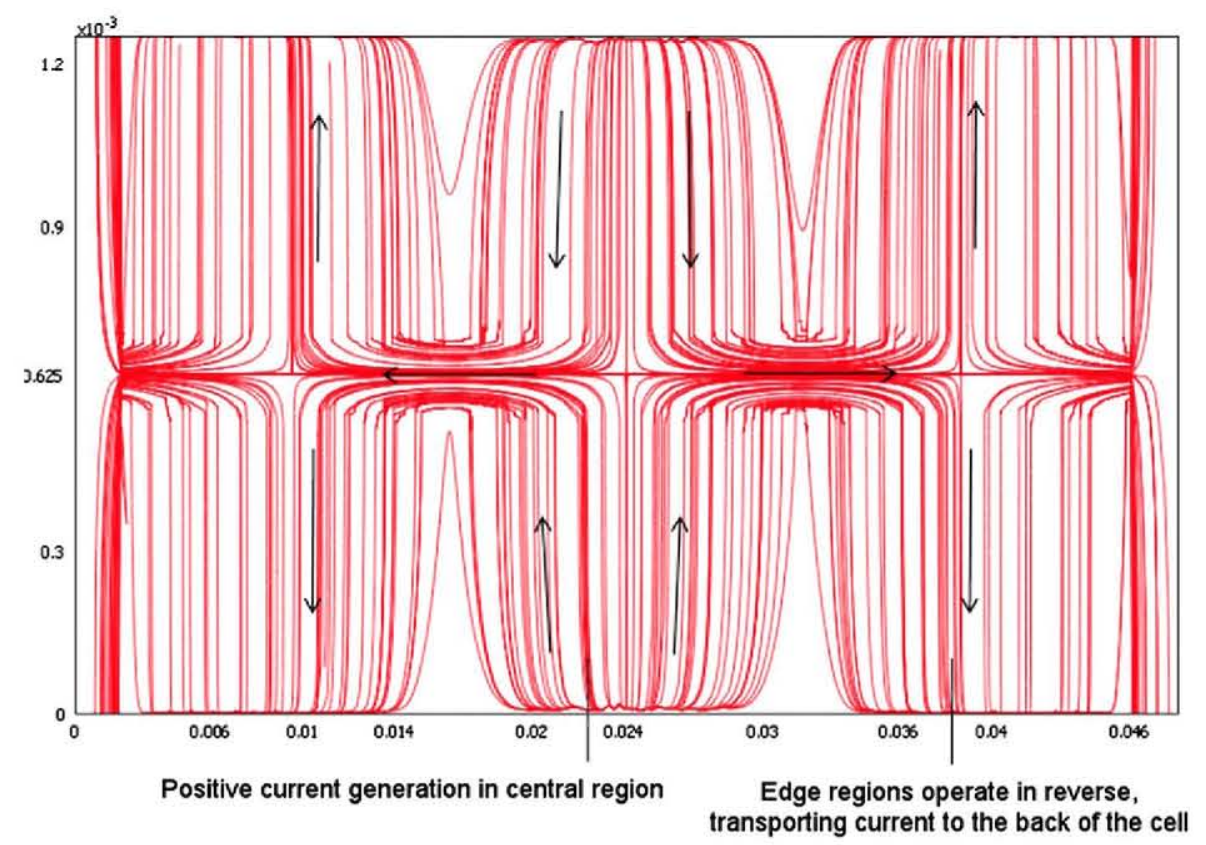

Fig. 8. Streamlines of current flow in the cell operating at open circuit voltage under non-uniform illumination. Arrows added to assist the reader show the direction of conventional current flow. 


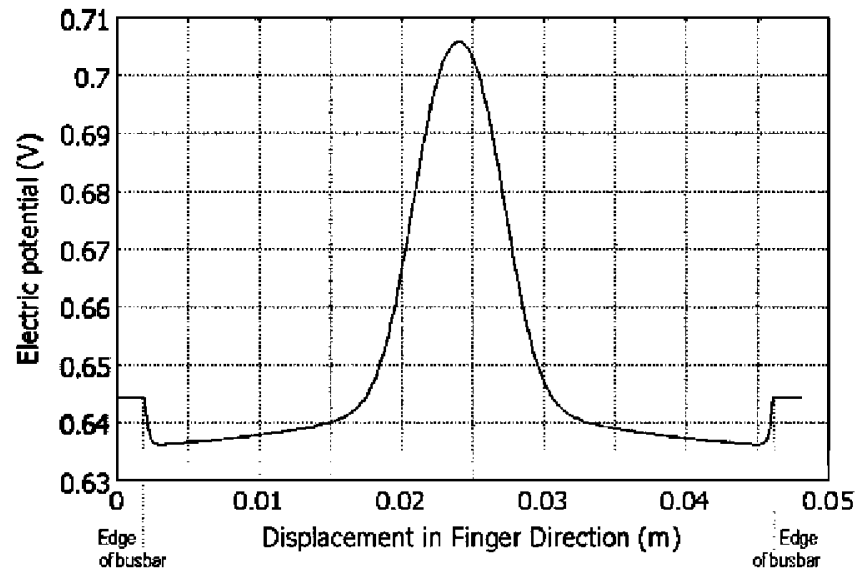

Fig. 9. A cross sectional plot of the spatial variance of the voltage in the finger direction. The cross section is taken at the upper edge of the element shown in Fig. 6.

dent on the local illumination intensity, which in conjunction with the local junction voltage dictates the current density generated at that point. Consequently, the darker regions of the cell have a lower local open circuit voltage than the lighter regions. Hence, although the voltage of the darker region is lower than that of the lighter region, the darker region operates above its local open circuit voltage, producing reverse current, and the lighter region operates below, producing forward current.

\section{Conclusions}

The presented model, which is experimentally verified, shows that cells operating under non-uniform Gaussian illumination experience a decrease in the fill factor, efficiency, and open circuit voltage, which correlate with the degree of non-uniformity of illumination.

A cell operating at open circuit region under Gaussian non-uniform illumination is shown to have internal currents which flow from the central highly illuminated regions of the emitter to the darker edge regions. This effect produces a partition of the cell into domains in which some (in the highly illuminated centre) behave as a current source, and others (in the weakly illuminated edge regions) behave as a drain, and has a significant detrimental impact on the maximum power point.

The efficiency of a cell operating under illumination with a peak illumination ratio of 10 and average illumination of 12 suns is shown to decrease by more than $1.7 \%$ when compared to a cell operating under a uniform illumination of 12 suns. It is shown that reoptimization of the front contact pattern, by increasing the number of fingers to suit the degree of non-uniformity, can mitigate the decrease in each characteristic significantly. For example, if the number of fingers on a cell operating under illumination with a peak illumination ratio of 10 is increased from 184 to 287 , the efficiency drop when compared to uniformly illuminated cell is decreased from over $1.7 \%$ to less than $0.7 \%$.

\section{Acknowledgements}

This work has been supported by Spanish Ministerio de Educación y Ciencia (MECI) under grant ENE2007-65410. We would like to thank the New and Renewable Energy Centre (NaREC) Photovoltaic Technology Centre (PVTC), Northumberland, UK, for sharing preliminary experimental data with us in order to assist in our modeling.

\section{References}

Cole, A., et al., 2006. Laser grooved buried contact solar cells for concentration factors up to $100 \times$. In: Conference Record of the 2006 IEEE 4th World Conference on Photovoltaic Energy Conversion, pp. 834-837.

Coventry, J.S., 2005. Performance of a concentrating photovoltaic/ thermal collector. Solar Energy 78, 211-222.

Coventry, J., Franklin, E., Blakers, A., 2004. Thermal and electrical performance of a concentrating PV/Thermal collector: results from the ANU CHAPS collector.

Franklin, E., Coventry, J., 2003. Effects of highly non-uniform illumination distribution on electrical performance of solar cells. In: Proceedings of Solar. Australian and New Zeeland Solar Energy Society. Available from: http://dspace-prod1.anu.edu.au/handle/1885/40832.

Galiana, B., Algora, C., Rey-Stolle, I., Garcia Vara, I., 2005. A 3-D model for concentrator solar cells based on distributed circuit units. IEEE Transactions on Electron Devices 52 (12), 2552-2558.

Johnston, G., 1998. Focal region measurements of the $20 \mathrm{~m}^{2}$ tiled dish at the Australian National University. Solar Energy 63, 117-124.

Luque, A., Hegedus, S., 2003. Handbook of Photovoltaic Science and Engineering. Wiley.

Luque, A., Sala, G., Arboiro, J., 1998. Electric and thermal model for non-uniformly illuminated concentration cells. Solar Energy Materials and Solar Cells 51, 269-290.

Rosell, J.I., Ibañez, M., 2006. Modelling power output in photovoltaic modules for outdoor operating conditions. Energy Conversion and Management 47, 2424-2430

van der Heide, A.S.H., Schönecker, A., Bultman, J.H., Sinke, W.C., 2005. Explanation of high solar cell diode factors by nonuniform contact resistance. Progress in Photovoltaics: Research and Applications 13, 316.

Zienkiewicz, O.C., Taylor, R.L., Zhu, J.Z., 2005. Finite Element Method: Its Basis and Fundamentals, sixth ed. Elsevier. 